Molecular Physics, 1986, Vol. 59, No. 3, 595-619

\title{
Stochastic descriptions of the dynamics of interacting brownian particles
}

\author{
by R. J. A. TOUGH and P. N. PUSEY \\ Royal Signals and Radar Establishment, St. Andrews Road, \\ Malvern, Worcestershire WR14 3PS England \\ H. N. W. LEKKERKERKER \\ Van't Hoff Laboratory, Rijksuniversiteit Utrecht, \\ Padualaan 8, 3508 TB Utrecht, The Netherlands \\ and C. VAN DEN BROECK \\ Limburgs Universitair Centrum, B-3610 Diepenbeek, Belgium
}

(Received 29 April 1986; accepted 4 Yune 1986)

\begin{abstract}
We review stochastic descriptions of the dynamics of colloidal particles, suspended in a liquid, which interact both directly and hydrodynamically. The equivalence of approaches based on Smoluchowski and Langevin equations is established. Particular attention is paid to the Itô and Stratonovich interpretations of stochastic differential equations with multiplicative noise. The short time behaviour of the correlation between two functions of particle position is discussed in some detail and compared with that found for a simple liquid. Finally the non-gaussian statistical properties of particle displacement are considered in the presence and absence of hydrodynamic interactions.
\end{abstract}

\section{Introduction}

There has been considerable interest in recent years in the proper theoretical description of the diffusive motions of colloidal particles suspended in a liquid at concentrations where the interparticle interactions, both direct and hydrodynamic, play an important role. Approaches based on Langevin and Smoluchowski equations have been used and there has been some discussion concerning the relationships between these various treatments. It does not appear to have penetrated fully the literature on particle suspensions that many of these questions have been extensively discussed and largely resolved in the literature on the theory of noise and stochastic processes.

In this paper we will concentrate on the Smoluchowski equation (i.e. the Fokker-Planck equation in co-ordinate space), which describes the evolution in time of the probability distribution of the particle positions, and the coupled Langevin equations which essentially generate trajectories of the particles. If hydrodynamic interactions are neglected the equivalence of these two approaches appears to be well established. However when hydrodynamic interactions are considered the Langevin equations are stochastic differential equations with 
multiplicative noise which, standing alone, are ambiguous or even meaningless, unless supplemented by an interpretative calculus such as those of Itô and Stratonovich. While these considerations are not completely absent in the suspension literature there appears to be no clear statement of their importance.

Thus this paper has several aims: firstly to clarify the issues involved and to emphasize the equivalence of the Smoluchowski and Langevin approaches; secondly to point out that hydrodynamic interactions in a particle suspension provide an important physical example of stochastic differential equations with multiplicative noise. A subsidiary aim is to demonstrate using both approaches the mechanics of the calculation of some dynamical properties of a particle suspension. One might question the value of pursuing different but equivalent theoretical methods. The advantage of the Smoluchowski equation is that it can be derived by an appealing and direct thermodynamic argument. An important reason for considering Langevin equations is that they provide the algorithm used in the growing field of brownian dynamics computer simulations. Furthermore calculations based on the Langevin approach are possibly more physically transparent. Also, for the calculations presented here as examples, namely short-time properties of the suspension, both approaches have been used.

Crucial to the validity of the Smoluchowski description of brownian motion in a particle suspension is the existence of a separation of timecales. Consider first an isolated particle (as in a very dilute suspension). Brownian motion of the particle is, of course, the result of the thermal agitation of the liquid molecules which cause the particle's velocity to fluctuate rapidly in magnitude and direction. The typical duration $\tau_{\mathbf{B}}$ of such a velocity fluctuation is roughly the ratio, $m /(6 \pi \eta a)$, of the particle's mass $m$ to its friction factor ( $\eta$ is the viscosity of the liquid and $a$ is the radius of the particle). For particles less than about one micron in size this time is typically less than one microsecond. Simple calculations show that in the time $\tau_{B}$ such a brownian particle moves a distance equal to only a very small fraction of its radius. In other words fluctuations in the particle's velocity relax on a timescale over which the particle hardly moves. Then, as is well known, the motion of the particle over times much longer than $\tau_{B}$ can be described in terms of a pure diffusion or random-walk process with mean-square displacement increasing linearly with time.

In a concentrated suspension, in which interparticle interactions are important, thermal agitations of the liquid still cause rapid fluctuations in the velocities of the individual particles. Furthermore fluctuations in the velocities of different particles become correlated through hydrodynamic interactions. Nevertheless we still expect that in a typical suspension the auto and cross correlations of the particle velocities created by the liquid agitation will decay over a time comparable to $\tau_{\mathbf{B}}$ during which the positions of the particles barely change. On a longer timescale, however, the particles will move significantly, the position-dependent forces between the particles will change as will their configuration-dependent hydrodynamic couplings.

Many experimental techniques, such as dynamic light scattering, are sensitive only to changes in the positions of the particles. Therefore a theoretical framework for interpreting such measurements only needs to describe accurately processes occurring over times much greater than $\tau_{\mathbf{B}}$. The Smoluchowski and overdamped Langevin equations are two such descriptions in which the rapid $\left(\leqslant \tau_{\mathrm{B}}\right)$ processes are treated in a coarse-grained stochastic (or diffusive) manner, 
whereas changes in spatial configuration are treated explicitly. Thus, as has been emphasized previously, these descriptions are designed to be valid for times $\tau \gg \tau_{B}$ but comparable to $\tau_{\mathrm{I}}$, the time required for a significant change in the spatial configuration of the particles.

In $\S 2$ we discuss the Langevin and Smoluchowski equations whereas $\S 3$ deals with calculations of the short time properties of a particle suspension. A general discussion and a more complete summary are given in $\$ 4$.

\section{SMOluchowski AND LANGEVIN EQUATIONS}

As outlined in the Introduction much of the discussion of the dynamics of interacting brownian particles in suspension is couched in terms of the probability that the particles adopt a given configuration at a given time. A formal development of an equation of motion for this probability - the so-called Kramers-Moyal expansion-can be undertaken [1]. A more physically motivated equation-the many particle Smoluchowski equation-was obtained [2] by generalizing the classic approach of Einstein [3]. The terms arising in the Smoluchowski equation can be identified with a subset of those in the general Kramers-Moyal expansion. A discussion of the Langevin equations or Stochastic Differential Equations (SDE) which are statistically equivalent to the Smoluchowski equation is then possible. This is relatively straightforward in the case in which the brownian fluctuations in different particles' positions can be taken to be uncorrelated. In physical terms this corresponds to the neglect of hydrodynamic interaction or solvent transmitted coupling of the particles' motion. The analogous description of the dynamics of particles in a suspension in which the hydrodynamic interaction cannot be neglected and the brownian fluctuations in the particles' positions depend on their configuration is not straightforward and requires more care. The formal description of such processes is well documented in the literature on noise and stochastic processes [4-7]; the insights afforded by this work allow us to demonstrate the consistency of several apparently different, Langevin treatments of suspension dynamics which have appeared in recent years [2, 8-11]. In carrying through the programme we have just outlined we also perform the useful pedagogical exercise of outlining the theory of SDE in a context in which their physical interpretation is familiar, and in which the principal results of the theory have already found practical application.

The temporal evolution of $\mathbf{P}\left(\mathbf{r}^{N}, t\right)$, the probability that the $N$ particles in the suspension adopt the configuration $\boldsymbol{r}^{N}\left(\left\{r_{1}, r_{2}, \ldots, r_{3 N}\right\}\right.$, their cartesian coordinates) at time $t$, can be described by the master equation [12]

$$
\mathbf{P}\left(\mathbf{r}^{N}, t+\Delta t\right)=\int \mathbf{P}\left(\mathbf{r}^{N}-\Delta^{N}, t\right) W\left(\mathbf{r}^{N}-\Delta^{N}, \Delta^{N}, \Delta t\right) d \mathbf{\Delta}^{N}
$$

This embodies the assumption that a description in terms of the particles positions is sufficiently complete on timescales of interest to be treated as a historyindependent, Markov process. $W\left(\mathbf{r}^{N}, \mathbf{\Delta}^{N}, \Delta t\right)$ is the probability distribution for changes $\Delta^{N}$ in the particles configuration, starting from a configuration $\mathbf{r}^{\prime}$, in 
time $\Delta t$. The dependence of $W$ on the initial configuration $\mathbf{r}^{\prime N}$ incorporates possible configuration dependence of the interparticle forces and the brownian fluctuations in the particle positions. It is straightforward to make a Taylor series expansion of the integrand in equation (2.1) to give

$$
\begin{aligned}
\frac{\partial \mathbf{P}\left(\mathbf{r}^{N}, t\right)}{\partial t} & =\lim _{\Delta t \rightarrow 0}\left(\frac{\mathbf{P}\left(\mathbf{r}^{N}, t+\Delta t\right)-\mathbf{P}\left(\mathbf{r}^{N}, t\right)}{\Delta t}\right) \\
& =\sum_{n=1}^{\infty} \frac{(-1)^{n}}{n !} \sum_{\substack{j_{1}, \ldots \\
j_{n}=1}}^{3 N} \frac{\partial}{\partial r_{j_{1}}} \cdots \frac{\partial}{\partial r_{j_{n}}}\left(a_{j_{1} \ldots j_{n}}\left(\mathbf{r}^{N}\right) \mathbf{P}\left(\mathbf{r}^{N}, t\right)\right)
\end{aligned}
$$

where

$$
a_{j_{1} \ldots j_{n}}\left(\mathbf{r}^{N}\right)=\lim _{\Delta t \rightarrow 0}\left\{\frac{1}{\Delta t} \int d \mathbf{\Delta}^{N} \Delta_{j_{1}} \ldots \Delta_{j_{n}} W\left(\mathbf{r}^{N}, \mathbf{\Delta}^{N}, \Delta t\right)\right\}
$$

and is assumed to be finite. The result (2.2) is known as the Kramers-Moyal expansion of the master equation. As it stands this represents no more than a re-expression of the Markov assumption implicit in equation (2.1). If it is assumed that the changes in particle configuration are small so that $W$ is a sharply peaked function of $\mathbf{\Delta}^{\boldsymbol{N}}$, but a slowly varying function of the initial configuration $\mathbf{r}^{N}$, and that $\mathbf{P}\left(\mathbf{r}^{N}, t\right)$ varies sufficiently smoothly for a Taylor series to be truncated, the form of equation (2.2) raises the expectation that a diffusive description of the particle dynamics will be appropriate [13].

To clarify the stochastic description of the dynamics of the suspension we now turn to the more physically motivated Smoluchowski equation of motion for $\mathbf{P}\left(\mathbf{r}^{N}, t\right)$. By considering the particles' overdamped motions in response to the interparticle forces and the gradient of the thermodynamic potential in the suspension, and requiring that the number of particles in suspension be conserved, Zwanzig [2] has shown that for interacting particles $\mathbf{P}\left(\mathbf{r}^{N}, t\right)$ satisfies the Smoluchowski equation

$$
\frac{\partial \mathbf{P}\left(\mathbf{r}^{N}, t\right)}{\partial t}=\sum_{i, j=1}^{3 N} \frac{\partial}{\partial r_{i}} D_{i j}\left(\mathbf{r}^{N}\right)\left[\beta \mathbf{P}\left(\mathbf{r}^{N}, t\right) \frac{\partial}{\partial r_{j}} U\left(\mathbf{r}^{N}\right)+\frac{\partial}{\partial r_{j}} \mathbf{P}\left(\mathbf{r}^{N}, t\right)\right] .
$$

Here $\beta=1 /\left(k_{\mathrm{B}} T\right)$ where $k_{\mathrm{B}}$ is Boltzmann's constant and $T$ is the absolute temperature, $U\left(r^{N}\right)$ is the interparticle potential and $D_{i j}\left(\mathbf{r}^{N}\right)$ is the generalized diffusion tensor. Equation (2.4) represents a generalization of the diffusion equation derived by Einstein [3] for non-interacting brownian particles. The generalized Einstein result (valid on a timescale long compared to the relaxation time $\tau_{B}$ discussed in $\S 1$ )

$$
D_{i j}=k_{\mathrm{B}} T b_{i j}
$$

relates $D_{i j}$ to the mobility matrix $b_{i j}$, which specifies a given particle's (coarsegrained) drift velocity $\bar{v}_{i}$ in response to forces $F_{j}$ acting on the particles in the suspension

$$
\bar{v}_{i}=\sum_{j=1}^{3 N} b_{i j} F_{j}
$$


We also note that the stationary solution of equation (2.4) is the Boltzmann distribution

$$
\mathbf{P}\left(\mathbf{r}^{N}, \infty\right)=\text { const. } \exp \left[-\beta U\left(\mathbf{r}^{N}\right)\right] .
$$

It is straightforward to show that the Smoluchowski equation (2.4) is of the standard Kramers-Moyal form, when the following identifications are made:

$$
\left.\begin{array}{rl}
a_{i} & =\sum_{j=1}^{3 N}\left(\frac{\partial}{\partial r_{j}} D_{i j}\left(\mathbf{r}^{N}\right)-\beta D_{i j}\left(\mathbf{r}^{N}\right) \frac{\partial}{\partial r_{j}} U\left(\mathbf{r}^{N}\right)\right) \\
a_{i j} & =2 D_{i j}\left(\mathbf{r}^{N}\right), \\
a_{j_{1} \ldots j_{n}} & =0, \quad n \geqslant 3 .
\end{array}\right\}
$$

A truncation of the Kramers-Moyal expansion at this level cannot be justified readily in any rigorous way, and so can hardly be taken to furnish a derivation of equation (2.4). Nonetheless the Smoluchowski equation itself has been deduced on the basis of standard physical arguments and provides a useful probabilistic description of the dynamics of particles in suspension.

An alternative, and appealing, stochastic description is provided by equations of motion for the particle co-ordinates themselves which generate trajectories including random contributions which may be identified with brownian fluctuations in position. In ensuring that the increments in these trajectories have the statistical properties attributed to the displacement terms $\Delta^{N}$ by equations $(2.3)$ and (2.8) (at least in the limit $\Delta t \rightarrow 0$ ) a random process is generated which is stochastically equivalent to that described by the Smoluchowski equation (2.4). Such trajectories can be constructed by exploiting simple properties of Gaussian random variables $[14]$. One sets

$$
\Delta_{i}=-\sum_{j=1}^{3 N}\left(\beta D_{i j}\left(\mathbf{r}^{N}\right) \frac{\partial}{\partial r_{j}} U\left(\mathbf{r}^{N}\right)-\frac{\partial}{\partial r_{j}} D_{i j}\left(\mathbf{r}^{N}\right)\right) \Delta t+R_{i}(\Delta t),
$$

where $R_{i}$ is chosen from a $3 N$-variate gaussian distribution such that

$$
\begin{aligned}
\overline{R_{i}(\Delta t)} & =0, \\
\overline{R_{i}(\Delta t) R_{j}(\Delta t)} & =2 D_{i j}\left(\mathbf{r}^{N}\right) \Delta t
\end{aligned}
$$

and all quantities are evaluated for the particle configuration prior to incrementation. From the well known properties of multivariate gaussian random variables it then follows that the $\Delta_{i}$ have the required statistical properties in the limit $\Delta t \rightarrow 0$. This is equivalent to $W\left(r^{\prime N}, \Delta^{N}, \Delta t\right)$ having a multivariate gaussian form at very short times and is consistent with our subsequent finding that non-gaussian statistical properties of particle displacements are manifest at order $(\Delta t)^{2}$ or higher (see $\S 3.5$ ). On taking $\Delta t$ to be small but non-zero the process (2.9) models that described by the Smoluchowski equation, to an accuracy of order $\Delta t$, and forms the basis of the widely used brownian dynamics algorithm of Ermak and $\mathrm{McCammon}$ [9]. The first contribution to the displacement $\Delta_{i}$ in equation (2.9) is commonly called the drift term whereas the second term is frequently referred to as the diffusion or brownian contribution.

As the process (2.9) is only a numerical approximation to that described by the Smoluchowski equation, the question arises as to whether it is possible, formally, to take the limit $\Delta t \rightarrow 0$ and obtain a differential, rather than a discrete equation of 
motion. We consider first the case in which hydrodynamic interactions can be neglected for which equation (2.9) reduces, on setting

$$
D_{i j}=D \delta_{i j}
$$

to

$$
\Delta_{i}=\beta D F_{i}\left(\mathbf{r}^{N}\right) \Delta t+R_{i}(\Delta t),
$$

where $D$ is the free-particle diffusion constant,

$$
F_{i}\left(\mathbf{r}^{N}\right)=-\frac{\partial}{\partial r_{i}} U\left(\mathbf{r}^{N}\right)
$$

and

$$
\overline{R_{i}(\Delta t) R_{j}(\Delta t)}=2 D \Delta t \delta_{i j} .
$$

We rewrite equation (2.12) in the form

$$
\frac{\Delta_{i}}{\Delta t}=\beta D F_{i}\left(\mathbf{r}^{N}\right)+\frac{R_{i}(\Delta t)}{\Delta t} .
$$

An estimate of the magnitude of the second term on the right hand side of equation (2.14) is provided by $\left[\left(\overline{\left.R_{i}(\Delta t)\right)^{2}}\right]^{1 / 2} / \Delta t\right.$, which is $0\left((\Delta t)^{-1 / 2}\right)$ and diverges as $\Delta t \rightarrow 0$. This seemingly paradoxical result arises from our taking the $\Delta t \rightarrow 0$ limit of a description which is only valid for times long compared with the relaxation time $\tau_{\mathrm{B}}$ of brownian fluctuations (see $\S 1$ ). A formal construction which overcomes this difficulty is the Wiener process $W(t)[15,16]$. This is a nonstationary gaussian process, defined only for $t>0$, which has a mean and autocorrelation function given by

$$
\begin{aligned}
\overline{W(t)} & =0 \\
\overline{W\left(t_{1}\right) W\left(t_{2}\right)} & =2 \min \left(t_{1}, t_{2}\right),
\end{aligned}
$$

where $\min \left(t_{1}, t_{2}\right)$ is the lesser of $t_{1}$ and $t_{2}$. The increment in $W(t)$ between times $t$ and $t+d t$ is termed the Wiener measure $d W(t)$ and the paradox implicit in equation (2.14) is resolved by decomposing the infinitesimal increment $d r_{i}(t)$ into two contributions

$$
d r_{i}(t)=\beta D F_{i}\left(\mathbf{r}^{N}(t)\right) d t+D^{1 / 2} d W(t) .
$$

This provides a simple example of a stochastic differential equation [17]. An integration to first order in $\Delta t$ gives us

$$
\Delta_{i}=r_{i}(t+\Delta t)-r_{i}(t)=\beta D F_{i}\left(\mathbf{r}^{N}(t)\right) \Delta t+D^{1 / 2}[W(t+\Delta t)-W(t)],
$$

which we see has the same form as equation (2.12). At this point it may appear that the discussion has become circular, or at least casuistical. However (though we will not pursue such matters here) the concept of the Wiener measure does make possible a rigorous mathematical treatment of diffusive processes [18]. Contact with physical intuition can be made by identifying (in a heuristic way) the derivative of the Wiener process with the gaussian white noise process $f(t)$

$$
\frac{d W_{i}(t)}{d t}=f_{i}(t)
$$


The properties (2.15) and (2.16) of the Wiener process then follow from those customarily assigned to the gaussian white noise process

$$
\begin{aligned}
\overline{f_{i}(t)} & =0, \\
\overline{f_{i}\left(t^{\prime}\right) f_{j}\left(t^{\prime \prime}\right)} & =2 \delta\left(t^{\prime}-t^{\prime \prime}\right) \delta_{i j}
\end{aligned}
$$

and a few formal manipulations. We also note that iterative integration of equation (2.17) to yield higher order expansions of $\Delta_{i}$ has been carried out, in both the context of dynamics of suspensions $[10,11]$ and of stochastic differential equations in general [19]. The identification (2.19) allows the physically appealing, but mathematically appalling, gaussian white noise to be contained within a rigorous mathematical structure. We may now also rewrite equation (2.17) in a more familiar Langevin form as

$$
\frac{d r_{i}}{d t}=\beta D F_{i}\left(\mathbf{r}^{N}\right)+D^{1 / 2} f_{i}(t)
$$

The mathematically well-defined equation (2.17) and its more intuitive counterpart equation (2.22) constitute stochastic descriptions which are fully equivalent to the Smoluchowski equation without hydrodynamic interactions (obtained by using equation (2.11) in equation (2.4)). Equation (2.22) allows one to interpret the particle's velocity as the sum of two contributions; the drift velocity in response to interparticle forces $\left(v_{1}\right.$ of [10]) and the brownian velocity caused by the buffeting of the suspending fluid $\left(v_{B}\right.$ of [10]). Although this separation into two components is physically appealing one should be wary of writing Langevin type equations ad hoc. Generally the intuitive augmentation of a deterministic equation of motion by a white noise term is an uncontrolled description of a physical system [20]. This becomes particularly clear when we turn to the case in which hydrodynamic interactions are not neglected.

In this situation the discrete, but approximate equation of motion (2.9) remains valid and is, in the limit $\Delta t \rightarrow 0$ equivalent to the Smoluchowski equation. However the formal passage to this limit and the recovery of a Langevin equation are more problematic. If, by analogy with equation $(2.22)$ we write down the equation of motion

$$
\frac{d r_{i}}{d t}=-\sum_{j=1}^{3 N}\left(\beta D_{i j}\left(\mathbf{r}^{N}\right) \frac{\partial}{\partial r_{j}} U\left(\mathbf{r}^{N}\right)-\frac{\partial}{\partial r_{j}} D_{i j}\left(\mathbf{r}^{N}\right)\right)+\sum_{j=1}^{3 N} \sigma_{i j}\left(\mathbf{r}^{N}\right) f_{j}(t),
$$

where $f_{j}(t)$ are white noise terms defined by the properties (2.20) and (2.21) and $\sigma_{i j}\left(\mathbf{r}^{N}\right)$ satisfies

$$
D_{i j}\left(\mathbf{r}^{N}\right)=\sum_{k} \sigma_{i k}\left(\mathbf{r}^{N}\right) \sigma_{j k}\left(\mathbf{r}^{N}\right)
$$

we have, as we shall now argue, merely generated an ambiguous set of symbols. The noise term in equation (2.23) is uncorrelated over all times, no matter how short (see equation (2.21)). Furthermore $r_{i}$ is assumed to respond to this driving force, whose amplitude is seen to depend on $\boldsymbol{r}^{N}$. However with no continuity in $f_{i}(t)$, the value of $\mathbf{r}^{N}$ appropriate to the determination of the amplitude of the noise terms is not defined by equation (2.23). In particular equation (2.23) cannot, as it stands, be taken to imply equation (2.9) unambiguously on integration over a short period of time. This difficulty can be resolved in either of two ways, formally or by appeal to physical intuition. Unfortunately these two resolutions do 
not necessarily coincide. To avoid cumbersome notation we will consider for the moment the integration of the one-dimensional stochastic differential equation

$$
d x=g(x) d t+b(x) d W(t) .
$$

(The extension to many dimensions is obvious and will be made without further comment shortly.) Here the problem reduces to that of attributing some meaning to the integrals

$$
x(t)-x(0)=\int_{0}^{t} g\left(x\left(t^{\prime}\right)\right) d t^{\prime}+\int_{0}^{t} b\left(x\left(t^{\prime}\right)\right) d W\left(t^{\prime}\right) .
$$

The first of these integrals can be defined as the limit of a sum in the usual Riemann sense [21]; a formal definition or interpretation of the second integral as the limit of a sum, i.e.

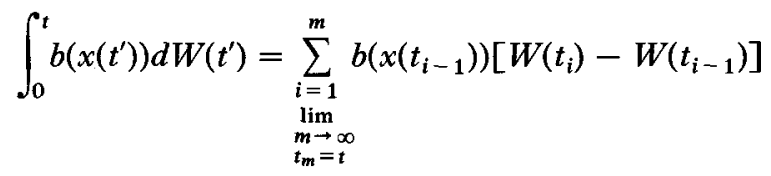

removes the ambiguity inherent in equation (2.25) and forms the basis of a rigorous mathematical theory of stochastic differential equations. This definition (2.27) of the integral, in which $b(x(t))$ is evaluated at the start of each time interval, is known as the Itô interpretation [22].

The physically motivated resolution of the difficulty is due to Stratonovich [23], who considers equation (2.25) in its more naive form

$$
\frac{d x}{d t}=g(x)+b(x) f(t)
$$

but takes $f(t)$ to be a continuous process with a non-zero correlation time. Equation (2.28) can then be treated as an ordinary differential equation (see the detailed discussions in [24] and [25]). The delta correlated white noise can be recovered at the end of the calculation by allowing the correlation time of $f(t)$ to tend to zero. This argument leads to an alternative interpretation of the second integral on the right hand side of equation (2.26) as the limit of a different sum, i.e.

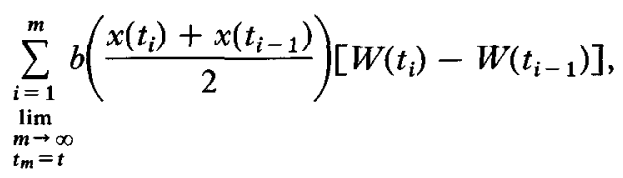

where $b(x)$ is evaluated at the mid-point of each time interval over which the Wiener process is evaluated. This reflects the continuity in the noise process inherent in its correlation: the system can 'anticipate' the behaviour of the noise modelled ultimately by the Wiener process. As the physical processes modelled by noise terms in Langevin equations must, when considered on a short enough timescale, be continuous the Stratonovich treatment has been claimed to give a more faithful description of reality [26]. Furthermore the Stratonovich treatment preserves the conventional rule of differential calculus in nonlinear transformations of SDE. In contrast the Itô interpretation implies rules for the nonlinear transformation of SDE which differ from those of ordinary calculus. Unfor- 
tunately a definition of a stochastic integral such as equation (2.29) is much more difficult to incorporate within a rigorous mathematical framework than the Itô interpretation (2.27), so that the latter forms the basis of much of the more detailed treatment of SDE occurring in the mathematical literature [16, 27].

The Itô and Stratonovich interpretations of the SDE (2.25) imply different values for the moments $a_{j_{1}} \ldots j_{n}$ in equation (2.3). The Itô case is straightforward and leads to

$$
\left.\begin{array}{l}
a_{1}=\lim _{\Delta t \rightarrow 0} \frac{\overline{\Delta x}}{\Delta t}=g(x), \\
a_{2}=\lim _{\Delta t \rightarrow 0} \frac{\overline{(\Delta x)^{2}}}{\Delta t}=2 b^{2}(x),
\end{array}\right\}
$$

with all higher moments vanishing so that equation $(2.25)$ is equivalent to a Fokker-Planck equation of the form

$$
\frac{\partial \mathbf{P}(x, t)}{\partial t}=-\frac{\partial}{\partial x}[g(x) \mathbf{P}(x, t)]+\frac{\partial^{2}}{\partial x^{2}}\left[b^{2}(x) \mathbf{P}(x, t)\right]
$$

The integration of equation (2.25) within the Stratonovich interpretation is more complicated as $W\left(t_{i}\right)-W\left(t_{i-1}\right)$ and

$$
b\left[\frac{x\left(t_{i}\right)+\cdots\left(t_{i-1}\right)}{2}\right]
$$

are not independent quantities. Consequently, to obtain terms to order $\Delta t$, the stochastic integral has to be iterated

$$
\begin{aligned}
\Delta x= & x(t+\Delta t)-x(t) \\
= & g(x(t)) \Delta t+b\left[\frac{x(t)+x(t+\Delta t)}{2}\right][W(t+\Delta t)-W(t)] \\
= & g(x(t)) \Delta t \\
& +\left\{b(x(t))+\frac{1}{2} \frac{d b(x)}{d x}[g(x(t)) \Delta t+b(x(t))[W(t+\Delta t)-W(t)]+\ldots]\right\} \\
& \times[W(t+\Delta t)-W(t)] .
\end{aligned}
$$

Upon averaging one obtains, using equations (2.15) and (2.16),

$$
\begin{aligned}
& a_{1}(x)=g(x)+b(x) \frac{d}{d x} b(x) \\
& a_{2}(x)=2 b^{2}(x)
\end{aligned}
$$

so that the Fokker-Planck equation equivalent to equation (2.25) interpreted in accord with the Stratonovich prescription is

$$
\frac{\partial \mathbf{P}(x, t)}{\partial t}=-\frac{\partial}{\partial x}\left[\left(g(x)+b(x) \frac{d}{d x} b(x)\right) \mathbf{P}(x, t)\right]+\frac{\partial^{2}}{\partial x^{2}}\left[b^{2}(x) \mathbf{P}(x, t)\right] .
$$

The above equation is manifestly different from equation (2.31) obtained using the Itô prescription.

Thus we see that a given Langevin equation which incorporates multiplicative noise is equivalent to two different Fokker-Planck equations, depending on 
whether it is interpreted according to the Itô or Stratonovich prescription. Conversely a given Fokker-Planck equation is equivalent to two different SDEs, each with its own different and well defined interpretation. There is obviously room for considerable confusion in this state of affairs, which has prompted a detailed discussion in the literature of stochastic processes. This has been reviewed extensively by several authors [5, 7, 28, 29] and more caustically by Van Kampen [6].

Within the context of the description of brownian motions coupled by hydrodynamic interaction it is imperative that a set of coupled Langevin equations, with a well defined prescription for their interpretation, be equivalent to the Smoluchowski equation (2.4) and, on integration to first order in $\Delta t$, yield the brownian dynamics algorithm (2.9). Bearing this in mind we now consider the various Langevin equations proposed in the literature to describe interacting brownian particles.

Ermak and McCammon [9] proposed the brownian dynamics algorithm in its discrete form (2.9) which is independent of any interpretative prescription and is equivalent to the Smoluchowski equation as $\Delta t \rightarrow 0$. Pusey and Tough have considered Langevin equations of the form (2.22) [10] and (2.23) [11]. In the former case the noise term is additive and no problem of interpretation arises. In the latter case the Langevin equation must be interpreted within the Itô convention if it is to be stochastically equivalent to the Smoluchowski equation. The formal manipulations of equation (2.23) performed in [11] are consistent with the Itô prescription, though this was not stated explicitly.

Hess and Klein [8] have obtained a Langevin equation for particle positions only, by a reduction of that describing positions and momenta. By considering the large friction limit, in which the correlation time for brownian fluctuations in velocities tend to zero, these authors are carrying through an analysis equivalent to that of Stratonovich. Consequently their result

$$
\frac{d r_{i}}{d t}=-\sum_{j=1}^{3 N} \beta D_{i j} \frac{\partial U}{\partial r_{j}}+\sum_{m, j=1}^{3 N} \sigma_{i m} \frac{\partial \sigma_{m j}}{\partial r_{j}}+\sum_{j=1}^{3 N} \sigma_{i j} f_{j}(t)
$$

is equivalent to the Smoluchowski equation only when interpreted within the Stratonovich convention. Finally we note that the Langevin equation, written down by $Z$ wanzig [2] to be equivalent to the Smoluchowski equation, is also to be interpreted in the Stratonovich sense. The apparent difference between this result and that of Hess and Klein [8], is due to Zwanzig's working with the divergenceless Oseen tensor when describing hydrodynamic interactions; this point is elaborated upon further in the Appendix.

\section{Short-time behaviour of a Particle Suspension}

In the previous section we discussed the equivalence of the Smoluchowski and Langevin descriptions of the dynamics of interacting brownian particles. In this section, as an example of the application of the formalism, we show how shorttime properties of a particle suspension can be calculated using both approaches. In $\$ 3.1$ we obtain from the Smoluchowski equation a general expression for the expansion in powers of time of the time correlation function of two arbitrary functions $f_{1}\left(\mathbf{r}^{N}\right)$ and $f_{2}\left(\mathbf{r}^{N}\right)$ of the particle configuration $\mathbf{r}^{N}$. In $\S 3.2$ the same general result is derived from the Langevin equation. In $\S 3.3$ we write down 
explicitly the first few terms in the expansions of a particle's mean-square displacement and the full and self-intermediate scattering functions, which can be measured by quasi-elastic (light or neutron) scattering methods. Section 3.4 explores the relationship between the dynamics of particles in suspension and of atoms in a simple liquid. Finally in $\S 3.5$ we discuss the statistical properties of the displacement of a particle and show that because of hydrodynamic interactions non-gaussian effects are found at shorter times in a particle suspension than is the case for atoms in a liquid.

\subsection{Short-time behaviour from the Smoluchowski equation}

We consider time correlation functions of the type

$$
F(\tau)=\left\langle f_{1}\left(\mathbf{r}^{N}(0)\right) f_{2}\left(\mathbf{r}^{N}(\tau)\right)\right\rangle
$$

where $f_{1}$ and $f_{2}$ are arbitrary functions of the particle positions $\mathbf{r}^{N}$ and the angular brackets indicate the configurational average

$$
\langle\cdot\rangle=\int d \mathbf{r}^{N} \int d \mathbf{r}_{0}^{N} \cdot \mathbf{P}\left(\mathbf{r}^{N}, \tau ; \mathbf{r}_{0}^{N}, 0\right),
$$

where $\mathbf{P}\left(\mathbf{r}^{N}, \tau ; \mathbf{r}_{0}^{N}, 0\right)$ is the probability that the particles adopt a configuration $\mathbf{r}_{0}^{N}$ $\left(\equiv \mathbf{r}^{N}(0)\right)$ at time $\tau=0$ and a configuration $\mathbf{r}^{N}\left(\equiv \mathbf{r}^{N}(\tau)\right)$ at time $\tau$. This can be expressed in terms of the conditional probability $\mathbf{P}\left(\mathbf{r}^{N}, \tau \mid \mathbf{r}_{0}^{N}, 0\right)$, defined by

$$
\mathbf{P}\left(\mathbf{r}^{N}, \tau ; \mathbf{r}_{0}^{N}, 0\right)=\mathbf{P}\left(\mathbf{r}^{N}, \tau \mid \mathbf{r}_{0}^{N}, 0\right) \mathbf{P}\left(\mathbf{r}_{0}^{N}\right),
$$

which can be regarded formally as the Green's function of the Smoluchowski equation (2.4). The one-time distribution is the canonical ensemble distribution

$$
P\left(\mathbf{r}_{0}^{N}\right)=\frac{\exp \left[-\beta U\left(\mathbf{r}_{0}^{N}\right)\right]}{Z},
$$

where $Z$ is the partition function. Thus we have

$$
F(\tau)=\frac{1}{Z} \int d \mathbf{r}^{N} \int d \mathbf{r}_{0}^{N} f_{1}\left(\mathbf{r}_{0}^{N}\right) f_{2}\left(\mathbf{r}^{N}\right) \mathbf{P}\left(\mathbf{r}^{N}, \tau \mid \mathbf{r}_{0}^{N}, 0\right) \exp \left[-\beta U\left(\mathbf{r}_{0}^{N}\right)\right] .
$$

The time evolution of the conditional probability $\mathbf{P}\left(\mathbf{r}^{N}, \tau \mid \mathbf{r}_{0}^{N}, 0\right)$ is given by the Smoluchowski equation (2.4) which we rewrite in the compact form

$$
\frac{\partial \mathbf{P}\left(\mathbf{r}^{N}, \tau \mid \mathbf{r}_{0}^{N}, 0\right)}{\partial \tau}=O_{\mathbf{r}} \mathbf{P}\left(\mathbf{r}^{N}, \tau \mid \mathbf{r}_{0}^{N}, 0\right)
$$

with initial condition

$$
\mathbf{P}\left(\mathbf{r}^{N}, 0 \mid \mathbf{r}_{0}^{N}, 0\right)=\delta\left(\mathbf{r}^{N}-\mathbf{r}_{0}^{N}\right) .
$$

The Smoluchowski operator

$$
O_{r}=\sum_{i, j=1}^{3 N} \frac{\partial}{\partial r_{i}} D_{i j}\left[\beta\left(\frac{\partial U}{\partial r_{j}}\right)+\frac{\partial}{\partial r_{j}}\right]
$$

acts on the variables $\boldsymbol{r}^{N}$. Equations (3.6) and (3.7) can be solved formally to give

$$
F(\tau)=\frac{1}{Z} \int d \mathbf{r}^{N} \int d \mathbf{r}_{0}^{N} f_{1}\left(\mathbf{r}_{0}^{N}\right) f_{1}\left(\mathbf{r}^{N}\right) \exp \left[-\beta U\left(\mathbf{r}_{0}^{N}\right)\right] \exp \left(O_{\mathbf{r}} \tau\right) \delta\left(\mathbf{r}^{N}-\mathbf{r}_{0}^{N}\right)
$$


An expansion of $F(\tau)$ in powers of $\tau$ can be calculated directly from equation (3.9) [30]. A more convenient procedure $[31,32]$ is based on the use of the adjoint operator

$$
\tilde{O}_{\mathbf{r}}=\sum_{i, j=1}^{3 N}\left[-\beta \frac{\partial U}{\partial r_{i}}+\frac{\partial}{\partial r_{i}}\right] D_{i j} \frac{\partial}{\partial r_{j}}
$$

Since

$$
\int d \mathbf{r}^{N} f_{2}\left(\mathbf{r}^{N}\right) \exp \left(O_{\mathbf{r}} \tau\right) \delta\left(\mathbf{r}^{N}-\mathbf{r}_{0}^{N}\right)=\int d \mathbf{r}^{N} \delta\left(\mathbf{r}^{N}-\mathbf{r}_{0}^{N}\right) \exp \left(\tilde{O}_{\mathbf{r}} \tau\right) f_{2}\left(\mathbf{r}^{N}\right)
$$

we can perform the integration over $\mathbf{r}^{N}$. This yields

$$
F(\tau)=\frac{1}{Z} \int d \mathbf{r}_{0}^{N} f_{1}\left(\mathbf{r}_{0}^{N}\right) \exp \left[-\beta U\left(\mathbf{r}_{0}^{N}\right)\right] \exp \left(\tilde{O}_{\mathbf{r}_{0}} \tau\right) f_{2}\left(\mathbf{r}_{0}^{N}\right)
$$

or, with a further contraction of notation $f_{1}\left(\mathbf{r}_{0}^{N}\right)=f_{1}$, etc,

$$
\begin{aligned}
F(\tau) & =\left\langle f_{1} \exp (\tilde{O} \tau) f_{2}\right\rangle \\
& =\sum_{n=0}^{\infty} \frac{\tau^{n}}{n !}\left\langle f_{1} \tilde{O}^{n} f_{2}\right\rangle .
\end{aligned}
$$

Note that such a short-time expansion is well-known in the theory of stochastic processes (see e.g. [33]). The adjoint Smoluchowski operator $\tilde{O}$ has the interesting property of being self-adjoint for the inner product defined by the ensemble averaging procedure, i.e.

$$
\langle f \tilde{O g}\rangle=\langle(\tilde{O f}) g\rangle
$$

as one easily verifies by partial integration. This property can be exploited for the explicit calculation of the terms in the expansion (3.13). Another relation which we will frequently use is

$$
\left\langle f\left(-\beta \frac{\partial U}{\partial r_{i}}+\frac{\partial}{\partial r_{i}}\right) g\right\rangle=-\left\langle\frac{\partial f}{\partial r_{i}} g\right\rangle
$$

which is direct consequence of the Yvon identity

$$
\beta\left\langle f \frac{\partial U}{\partial r_{i}}\right\rangle=\left\langle\frac{\partial f}{\partial r_{i}}\right\rangle
$$

We now calculate the first few terms in the expansion (3.13). For the zeroth order term, we obviously get

$$
\left\langle f_{1} \tilde{O}^{0} f_{2}\right\rangle=\left\langle f_{1} f_{2}\right\rangle .
$$

The $\tau$ term is given by

$$
\left\langle f_{1} \tilde{O f_{2}}\right\rangle=\sum_{i, j=1}^{3 N}\left\langle f_{1}\left(-\beta \frac{\partial U}{\partial r_{i}}+\frac{\partial}{\partial r_{i}}\right)\left(D_{i j} \frac{\partial f_{2}}{\partial r_{j}}\right)\right\rangle .
$$

Using equation (3.15) we get

$$
\left\langle f_{1} \tilde{O f_{2}}\right\rangle=-\sum_{i, j=1}^{3 N}\left\langle\frac{\partial f_{1}}{\partial r_{i}} D_{i j} \frac{\partial f_{2}}{\partial r_{j}}\right\rangle
$$


a result apparently first obtained by $Z$ wanzig [31]. For the second order term, we obtain, using the self-adjointness property (3.14)

$$
\begin{aligned}
\left\langle f_{1} \tilde{O}^{2} f_{2}\right\rangle & =\left\langle\left(\tilde{O} f_{1}\right)\left(\tilde{O} f_{2}\right)\right\rangle \\
& =\sum_{i, j, k, l=1}^{3 N}\left\langle\left[\left(-\beta \frac{\partial U}{\partial r_{i}}+\frac{\partial}{\partial r_{i}}\right) D_{i j} \frac{\partial}{\partial r_{j}} f_{1}\right]\left(-\beta \frac{\partial U}{\partial r_{k}}+\frac{\partial}{\partial r_{k}}\right) D_{k l} \frac{\partial}{\partial r_{l}} f_{2}\right\rangle .
\end{aligned}
$$

Again using equation (3.15) we now find that

$$
\begin{aligned}
\left\langle f_{1} \tilde{O}^{2} f_{2}\right\rangle=\sum_{i, j, k, l=1}^{3 N}\left\{\left\langle\left(\frac{\partial}{\partial r_{k}} D_{i j} \frac{\partial}{\partial r_{j}} f_{1}\right)\right.\right. & \left.\left(\frac{\partial}{\partial r_{i}} D_{k l} \frac{\partial}{\partial r_{l}} f_{2}\right)\right\rangle \\
& \left.+\beta\left\langle\frac{\partial f_{1}}{\partial r_{i}} D_{i j} \frac{\partial^{2} U}{\partial r_{j} \partial r_{k}} D_{k l} \frac{\partial f_{2}}{\partial r_{l}}\right\rangle\right\}
\end{aligned}
$$

A similar though increasingly tedious analysis can be applied to give the higher order terms.

\subsection{Short-time behaviour from the Langevin equation}

In order to obtain the result contained in equation (3.13) using the Langevin approach we first expand $f_{2}\left(\mathbf{r}^{N}(\tau)\right)$ in equation (3.1) as a Taylor series in particle displacements $\Delta_{i}$

$$
f_{2}\left(\mathbf{r}^{N}(\Delta t)\right)=f_{2}\left(\mathbf{r}^{N}(0)\right)+\left.\sum_{i=1}^{3 N} \frac{\partial f_{2}}{\partial r_{i}}\right|_{0} \Delta_{i}+\left.\frac{1}{2} \sum_{i, j=1}^{3 N} \frac{\partial^{2} f_{2}}{\partial r_{i} \partial r_{j}}\right|_{0} \Delta_{i} \Delta_{j}+\ldots
$$

The above equation describes the temporal evolution of the stochastic variable $f_{2}\left(\mathbf{r}^{N}(t)\right)$ from its value $f_{2}\left(\mathbf{r}^{N}(0)\right)$ for a given initial configuration $\boldsymbol{r}^{N}(0)$. We now perform an average of $f_{2}\left(r^{N}(\Delta t)\right)$ over the distribution of random displacements $\Delta_{i}$ for this given initial configuration. By using equations (2.9) and (2.10) we get

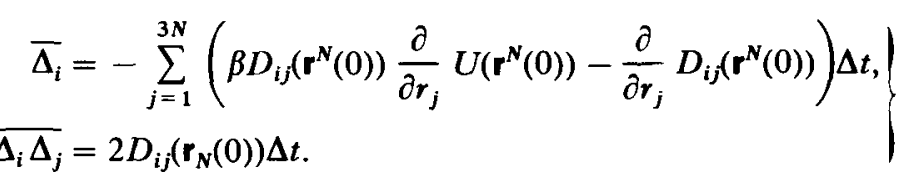

Note that to order $\Delta t$ the average $\bar{\Delta}_{i}$ comes from the drift terms whereas $\overline{\Delta_{i} \Delta_{j}}$ originates from the brownian displacements. The drift terms contribute to $\overline{\Delta_{i} \Delta_{j}}$ only to order $(\Delta t)^{2}$. Taking the indicated average of equation (3.19) and using the results given by equation (3.20) we find

$$
\overline{f_{2}\left(\mathbf{r}^{N}(\Delta t)\right)}=(1+\tilde{O} \Delta t) f_{2}\left(\mathbf{r}^{N}(0)\right)
$$

where $\tilde{O}$ is the adjoint Smoluchowski operator defined by equation (3.10). Iterating equation (3.21) over $n$ short intervals $\Delta t$ then gives

$$
\overline{f_{2}\left(\mathbf{r}^{N}(n \Delta t)\right)}=(1+\tilde{O \Delta t})^{n} f_{2}\left(\mathbf{r}^{N}(0)\right)
$$

so that in the limit $\Delta t \rightarrow 0, n \rightarrow \infty, n \Delta t=\tau$ we get

$$
\overline{f_{2}\left(\mathbf{r}^{N}(\tau)\right)}=\exp (\tilde{O} \tau) f_{2}\left(\mathbf{r}^{\mathbf{N}}(0)\right) \text {. }
$$

Finally substitution of equation (3.22) in equation (3.1) gives

$$
F(\tau)=\left\langle f_{1} \exp (\tilde{O} \tau) f_{2}\right\rangle
$$


in agreement (not surprisingly in view of $\S 2$ ) with equation (3.13) derived from the Smoluchowski equation.

The earlier calculations of Pusey and Tough $[10,11]$ showed explicitly the separate averaging over brownian fluctuations and particle configurations, which we have just seen provide a direct route to the equations of motion of functions of particle positions generated by the adjoint Smoluchowski operator $\tilde{O}$. Thus it might be claimed that such Langevin based calculations are physically more transparent. However, it should be stressed that both the 'fast' averaging over particle displacements to yield the adjoint operator $\tilde{O}$ and a subsequent ensemble averaging over particle configurations are implicit in the more formal treatment based on the Smoluchowski equation. To see this explicitly we refer to the analysis in $\$ 3.1$. Here the integration of co-ordinates $\mathbf{r}^{N}$ over the conditional probability or propagator

$$
\mathbf{P}\left(\mathbf{r}^{N}, \tau \mid \mathbf{r}_{0}^{N}, 0\right)=\exp (O \tau) \delta\left(\mathbf{r}^{N}-\mathbf{r}_{0}^{N}\right)
$$

averages over particle displacements in a time $\tau$ from an initial configuration $\mathbf{r}_{0}^{N}$ and, through integration by parts over the delta function, introduces the adjoint operator $\tilde{O}$. This leaves just the final canonical ensemble average to be performed.

\subsection{Explicit results for the mean square displacement and intermediate scattering functions}

One cartesian component of the mean square displacement of a particle is given by

$$
\begin{aligned}
\left\langle\Delta_{i}^{2}(\tau)\right\rangle & =\left\langle\left[r_{i}(\tau)-r_{i}(0)\right]^{2}\right\rangle \\
& =2\left\langle r_{i}^{2}\right\rangle-2\left\langle r_{i}(0) r_{i}(\tau)\right\rangle .
\end{aligned}
$$

To calculate the time expansion of the above quantity we use the results (3.16)(3.18) with $f_{1}=f_{2}=r_{i}$. This gives

$$
\left\langle\Delta_{i}^{2}(\tau)\right\rangle=2\left\langle D_{i i}\right\rangle \tau-\tau^{2} \sum_{k, l=1}^{3 N}\left\{\beta\left\langle D_{i k} \frac{\partial^{2} U}{\partial r_{i} \partial r_{k}} D_{i l}\right\rangle+\left\langle\frac{\partial D_{i l}}{\partial r_{k}} \frac{\partial D_{i k}}{\partial r_{l}}\right\rangle\right\}+O\left(\tau^{3}\right) .
$$

For a suspension of $N$ identical brownian particles the full intermediate scattering function measured by quasi-elastic scattering techniques can be written as

$$
F(K, \tau)=N^{-1} \sum_{i, j=1}^{N}\left\langle\exp \left[i K\left(r_{i}(0)-r_{j}(\tau)\right)\right]\right\rangle .
$$

Here we have chosen the scattering vector $\mathbf{K}$ parallel to the $X$-axis and have denoted the $X$-components of the particles $1,2, \ldots, N$ by $r_{1}, r_{2}, \ldots, r_{N}$. To calculate the time-dependence of $F(K, \tau)$ we use the results of $\S 3.1$, taking

$$
f_{1}=\frac{1}{\sqrt{ } N} \sum_{j=1}^{N} \exp \left(i K r_{j}\right)
$$

and

$$
f_{2}=\frac{1}{\sqrt{ } N} \sum_{j=1}^{N} \exp \left(-i K r_{j}\right)
$$


Thus, for example

$$
\begin{aligned}
\frac{\partial f_{1}}{\partial r_{j}} & =\frac{i K}{\sqrt{ } N} \exp \left(i K r_{j}\right), \quad 1 \leqslant j \leqslant N \\
& =0 \text { otherwise }
\end{aligned}
$$

and

$$
\begin{aligned}
\frac{\partial^{2} f_{1}}{\partial r_{j} \partial r_{l}} & =-\frac{K^{2}}{\sqrt{ } N} \exp \left(i K r_{j}\right) \delta_{j l}, \quad 1 \leqslant j, l \leqslant N \\
& =0 \text { otherwise. }
\end{aligned}
$$

Proceeding in this manner gives

$$
\begin{aligned}
F(K, \tau)= & S(K)-\frac{K^{2} \tau}{N} \sum_{j, l=1}^{N}\left\langle D_{j l} \exp \left(i K r_{j l}\right)\right\rangle \\
& +\frac{\tau^{2}}{2}\left\{\frac{K^{2}}{N} \sum_{j, l=1}^{N} \sum_{m, n=1}^{3 N}\left\langle\left[\beta D_{j m} D_{l n} \frac{\partial^{2} U}{\partial r_{m} \partial r_{n}}+\frac{\partial D_{j m}}{\partial r_{n}} \frac{\partial D_{l n}}{\partial r_{m}}\right] \exp \left(i K r_{j l}\right)\right\rangle\right. \\
& -\frac{2 K^{3}}{N} \sum_{j, l=1}^{N} \sum_{m=1}^{3 N}\left\langle D_{j m} \frac{\partial D_{j l}}{\partial r_{m}} \sin \left(K r_{j l}\right)\right\rangle \\
& \left.+\frac{K^{4}}{N} \sum_{j, l=1}^{N}\left\langle D_{j l}^{2} \exp \left(i K r_{j l}\right)\right\rangle\right\}+O\left(\tau^{3}\right)
\end{aligned}
$$

where the static structure factor $S(K)$ is given by

and

$$
S(K)=F(K, 0)
$$

$$
r_{j l}=r_{j}-r_{l} \text {. }
$$

The result for the self intermediate scattering function

$$
F_{s}(K, \tau)=\left\langle\exp \left[i K\left(r_{j}(0)-r_{j}(\tau)\right)\right]\right\rangle
$$

may be obtained either by using the results of $\S 3.1$ or simply by setting $j=l$ in equation (3.30). This gives

$$
\begin{aligned}
& F_{\mathrm{s}}(K, \tau)=1-K^{2} \tau\left\langle D_{11}\right\rangle \\
& +\frac{\tau^{2}}{2}\left\{K^{2} \sum_{m, n=1}^{3 N}\left\langle\beta D_{1 m} D_{1 n} \frac{\partial^{2} U}{\partial r_{m} \partial r_{n}}+\frac{\partial D_{1 m}}{\partial r_{n}} \frac{\partial D_{1 n}}{\partial r_{m}}\right\rangle+K^{4}\left\langle D_{11}^{2}\right\rangle\right\}+O\left(\tau^{3}\right) .
\end{aligned}
$$

Equations (3.24), (3.30) and (3.34) were quoted without derivation in [11].

\subsection{Comparison with simple liquids}

Formal similarities between the time expansions of correlation functions for a particle suspension in which hydrodynamic interactions can be neglected and the equivalent expressions for a simple atomic liquid have been noted by several authors $[30,34,35]$. For a liquid the analogue of the Smoluchowski equation is the Liouville equation and the analogue of the Langevin equation is Newton's second law of motion. For simplicity we consider only the latter approach here although again the two approaches can, of course, be shown to be equivalent. 
Liouville's and Newton's equations describe a classical liquid exactly. No coarse graining has been applied and it is necessary to assume that the function $f_{2}$ depends both on atomic positions and velocities, i.e. $f_{2}=f_{2}\left(\mathbf{r}^{N}(t), \mathbf{v}^{N}(t)\right)$. Thus

$$
f_{2}(\Delta t)=f_{2}(0)+\sum_{k=1}^{3 N}\left\{\left.\frac{\partial f_{2}}{\partial r_{k}}\right|_{0} v_{k}+\left.\frac{\partial f_{2}}{\partial v_{k}}\right|_{0} \frac{d v_{k}}{d t}\right\} \Delta t,
$$

which, with use of Newton's law

$$
\frac{d v_{k}}{d t}=-\frac{1}{m} \frac{\partial U}{\partial r_{k}}
$$

becomes

$$
f_{2}(\Delta t)=(1-\Delta t \mathscr{L}) f_{2}(0),
$$

where the Liouville operator $\mathscr{L}$ is given by

$$
\mathscr{L}=-\sum_{k=1}^{3 N}\left[v_{k} \frac{\partial}{\partial r_{k}}-\frac{1}{m} \frac{\partial U}{\partial r_{k}} \frac{\partial}{\partial v_{k}}\right] .
$$

On iterating equation (3.37) we get the well known result

$$
f_{2}(\tau)=\exp (-\mathscr{L} \tau) f_{2}(0)
$$

so that

$$
\begin{aligned}
F(\tau) & =\left\langle f_{1}(0) f_{2}(\tau)\right\rangle \\
& =\sum_{n=0}^{\infty} \frac{(-\tau)^{n}}{n !}\left\langle f_{1} \mathscr{L}^{n} f_{2}\right\rangle
\end{aligned}
$$

We note that $-\mathscr{L}$ is the adjoint of $\mathscr{L}$ for the inner product defined by the canonical ensemble averaging procedure, i.e.

$$
\langle f \mathscr{L} g\rangle=\langle(-\mathscr{L} f) g\rangle
$$

as can be verified by partial integration.

It is now instructive to consider the first few terms in equation (3.40) for the case of interest in this paper where $f_{1}$ and $f_{2}$ are explicit functions of particle configuration $\mathbf{r}^{N}(\tau)$ only. This means that the time correlation functions considered here are even functions of $\tau$ and therefore the coefficients of odd powers of $\tau$ in equation (3.40) are zero.

The $\tau^{2}$ term in equation (3.40) can easily be obtained by invoking the antihermitian property $(3.41)$ of $\mathscr{L}$

$$
\left\langle f_{1} \mathscr{L}^{2} f_{2}\right\rangle=-\left\langle\left(\mathscr{L} f_{1}\right)\left(\mathscr{L} f_{2}\right)\right\rangle .
$$

Since $f_{1}$ and $f_{2}$ are taken to be functions of the particle configurations only we find

$$
\begin{aligned}
\left\langle f_{1} \mathscr{L}^{2} f_{2}\right\rangle & =-\sum_{k, l=1}^{3 N}\left\langle v_{k} \frac{\partial f_{1}}{\partial r_{k}} v_{l} \frac{\partial f_{2}}{\partial r_{l}}\right\rangle \\
& =-\frac{k_{\mathrm{B}} T}{m} \sum_{k=1}^{3 N}\left\langle\frac{\partial f_{1}}{\partial r_{k}} \frac{\partial f_{2}}{\partial r_{k}}\right\rangle
\end{aligned}
$$


where we have averaged over velocities and have used the equi-partition of energy

$$
\left\langle v_{k} v_{l}\right\rangle=\frac{k_{\mathrm{B}} T}{m} \delta_{k l}
$$

It will be convenient to rewrite the result $(3,43)$ (with use of the Yvon identity (3.15))

$$
\left\langle f_{1} \mathscr{L}^{2} f_{2}\right\rangle=\left\langle f_{1} M f_{2}\right\rangle
$$

where the operator $M$ is defined by

$$
M=\sum_{k=1}^{3 N}\left(\frac{k_{\mathrm{B}} T}{m} \frac{\partial^{2}}{\partial r_{k}^{2}}-\frac{1}{m} \frac{\partial U}{\partial r_{k}} \frac{\partial}{\partial r_{k}}\right)
$$

In view of the property (3.41) it is evident that $M$ is self-adjoint, i.e.

$$
\left\langle f_{1} M f_{2}\right\rangle=\left\langle\left(M f_{1}\right) f_{2}\right\rangle .
$$

The $\tau^{4}$ term in equation (3.40) is

$$
\begin{aligned}
\left\langle f_{1} \mathscr{L}^{4} f_{2}\right\rangle= & \left\langle\left(\mathscr{L}^{2} f_{1}\right)\left(\mathscr{L}^{2} f_{2}\right)\right\rangle \\
= & \sum_{i, j, k, l=1}^{3 N}\left\langle\left[\left(v_{i} v_{j} \frac{\partial^{2}}{\partial r_{i} \partial r_{j}}-\frac{\delta_{i j}}{m} \frac{\partial U}{\partial r_{i}} \frac{\partial}{\partial r_{i}}\right) f_{1}\right]\right. \\
& \left.\times\left[\left(v_{k} v_{l} \frac{\partial^{2}}{\partial r_{k} \partial r_{l}}-\frac{\delta_{k l}}{m} \frac{\partial U}{\partial r_{k}} \frac{\partial}{\partial r_{k}}\right) f_{2}\right]\right\rangle \\
= & \sum_{i, k=1}^{3 N}\left\langle\left[\left(\frac{k_{\mathrm{B}} T}{m} \frac{\partial^{2}}{\partial r_{i}^{2}}-\frac{1}{m} \frac{\partial U}{\partial r_{i}} \frac{\partial}{\partial r_{i}}\right) f_{1}\right]\right. \\
& \left.\times\left[\left(\frac{k_{\mathrm{B}} T}{m} \frac{\partial^{2}}{\partial r_{k}^{2}}-\frac{1}{m} \frac{\partial U}{\partial r_{k}} \frac{\partial}{\partial r_{k}}\right) f_{2}\right]\right\rangle \\
& +2 \sum_{i, k=1}^{3 N}\left\langle\left(\frac{k_{\mathrm{B}} T}{m}\right)^{2} \frac{\partial^{2} f_{1}}{\partial r_{i} \partial r_{k}} \frac{\partial^{2} f_{2}}{\partial r_{i} \partial r_{k}}\right\rangle
\end{aligned}
$$

where we have used equations $(3.41),(3.44)$ and the gaussian property

$$
\left\langle v_{i} v_{j} v_{k} v_{l}\right\rangle=\left(\frac{k_{\mathrm{B}} T}{m}\right)^{2}\left(\delta_{i j} \delta_{k l}+\delta_{i k} \delta_{j l}+\delta_{i l} \delta_{j k}\right)
$$

of the velocities. From equations (3.43) and (3.45) we have

$$
\left\langle f_{1} M f_{2}\right\rangle=-\frac{k_{\mathrm{B}} T}{m} \sum_{k=1}^{3 N}\left\langle\frac{\partial f_{1}}{\partial r_{k}} \frac{\partial f_{2}}{\partial r_{k}}\right\rangle
$$

so that, with use of equations (3.46) and (3.47), (3.48) can be written in the compact form

$$
\left\langle f_{1} \mathscr{L}^{4} f_{2}\right\rangle=\left\langle f_{1} M^{2} f_{2}\right\rangle-\frac{2 k_{\mathrm{B}} T}{m} \sum_{i}\left\langle\frac{\partial f_{1}}{\partial r_{i}} M \frac{\partial f_{2}}{\partial r_{i}}\right\rangle .
$$

We note that the last term on the right hand side of equation (3.51) is zero if either $f_{1}$ or $f_{2}$ is a linear function of the co-ordinates. In particular we have

$$
\left\langle\boldsymbol{r}_{i} \mathscr{L}^{\mathbf{4}} \boldsymbol{r}_{j}\right\rangle=\left\langle\boldsymbol{r}_{i} \boldsymbol{M}^{2} \boldsymbol{r}_{j}\right\rangle
$$


Higher order terms in an expansion of the correlation function $\left\langle r_{i}(0) r_{j}(\tau)\right\rangle$ can be calculated easily using the property

$$
\mathscr{L}^{2} r_{i}=-\frac{1}{m} \frac{\partial U}{\partial r_{i}}=M r_{i}
$$

Thus

$$
\left\langle r_{i} \mathscr{L}^{6} r_{j}\right\rangle=\left\langle r_{i} \mathscr{L}^{4}\left(M r_{j}\right)\right\rangle
$$

so that, with use of equation (3.51), we get

$$
\begin{aligned}
\left\langle r_{i} \mathscr{L}^{6} r_{j}\right\rangle & =\left\langle r_{i} M^{2}\left(M r_{j}\right)\right\rangle \\
& =\left\langle r_{i} M^{3} r_{j}\right\rangle .
\end{aligned}
$$

Similarly, we have

$$
\begin{aligned}
\left\langle r_{i} \mathscr{L}^{8} r_{j}\right\rangle & =\left\langle\left(\mathscr{L}^{2} r_{i}\right) \mathscr{L}^{4}\left(\mathscr{L}^{2} r_{j}\right)\right\rangle \\
& =\left\langle\left(M r_{i}\right) \mathscr{L}^{4}\left(M r_{j}\right)\right\rangle \\
& =\left\langle\left(M^{2} r_{i}\right)\left(M^{2} r_{j}\right)\right\rangle+2\left(\frac{k_{\mathrm{B}} T}{m}\right)^{2} \sum_{k, l=1}^{3 N}\left\langle\frac{\partial^{2}\left(M r_{i}\right)}{\partial r_{k} \partial r_{l}} \frac{\partial^{2}\left(M r_{j}\right)}{\partial r_{k} \partial r_{l}}\right\rangle \\
& =\left\langle r_{i} M^{4} r_{j}\right\rangle+2\left(\frac{k_{\mathrm{B}} T}{m^{2}}\right)^{2} \sum_{k, l=1}^{3 N}\left\langle\frac{\partial^{3} U}{\partial r_{i} \partial r_{k} \partial r_{l}} \frac{\partial^{3} U}{\partial r_{j} \partial r_{k} \partial r_{l}}\right\rangle .
\end{aligned}
$$

The adjoint Smoluchowski operator for a particle suspension in which hydrodynamic interactions are neglected is obtained by setting (cf. equation (2.11))

$$
D_{i j}=\frac{k_{\mathbf{B}} T}{\zeta} \delta_{i j}
$$

where $\zeta$ is the single particle friction coefficient, in equation (3.10) to give

$$
\tilde{O}=\sum_{k=1}^{3 N}\left(\frac{k_{\mathrm{B}} T}{\zeta} \frac{\partial^{2}}{\partial r_{k}^{2}}-\frac{1}{\zeta} \frac{\partial U}{\partial r_{k}} \frac{\partial}{\partial r_{k}}\right)
$$

The reason for introducing the operator $M$ for simple liquids is now evident: comparison of equations (3.46) and (3.58) shows that $M$ is identical to $\tilde{O}$ if the particle friction coefficient $\zeta$ is replaced by the atom's mass $m$. With this replacement we see that the coefficient of $\tau / 1$ ! in equation (3.13) for a particle suspension is identical to the coefficient of $\tau^{2} / 2$ ! in equation (3.40) for a liquid (see equation (3.45)). The coefficient of $\tau^{2} / 2$ ! for a particle suspension differs from the coefficient $\tau^{4} / 4$ ! for a liquid only through the second term in equation (3.51). For the special case $f_{1}=r_{i}, f_{2}=r_{j}$ the quantities $\left\langle r_{i} \tilde{O} r_{j}\right\rangle,\left\langle r_{i} \tilde{O}^{2} r_{j}\right\rangle$ and $\left\langle r_{i} \tilde{O}^{3} r_{j}\right\rangle$ for a suspension are, subject to the replacement of $\zeta$ by $m$, identical to the quantities $\left\langle r_{i} \mathscr{L}^{2} r_{j}\right\rangle,\left\langle r_{i} \mathscr{L}^{4} r_{j}\right\rangle$ and $\left\langle r_{i} \mathscr{L}^{6} r_{j}\right\rangle$ for a liquid; however a difference is found when the $\tau^{4} / 4$ ! suspension term, $\left\langle r_{i} \widetilde{O}^{4} r_{j}\right\rangle$, is compared with the $\tau^{8} / 8$ ! liquid term, $\left\langle r_{i} \mathscr{L}^{8} r_{j}\right\rangle$ (compare equation (3.13) with (3.56)). It is perhaps worthwhile writing 
down the mean-square displacements in each case. For a particle suspension without hydrodynamic interactions we calculate

$$
\begin{aligned}
\left\langle\Delta_{1}^{2}(\tau)\right\rangle= & 2 \tau \frac{k_{\mathrm{B}} T}{\zeta}-\tau^{2} \frac{k_{\mathrm{B}} T}{\zeta^{2}}\left\langle\frac{\partial^{2} U}{\partial r_{1}^{2}}\right\rangle+\frac{\tau^{3}}{3} \frac{k_{\mathrm{B}} T}{\zeta^{3}} \sum_{i=1}^{3 N}\left\langle\left(\frac{\partial^{2} U}{\partial r_{1} \partial r_{i}}\right)^{2}\right\rangle \\
& -\frac{\tau^{4}}{12} \frac{1}{\zeta^{4}}\left[\left(k_{\mathrm{B}} T\right)^{2} \sum_{i, j=1}^{3 N}\left\langle\left(\frac{\partial^{3} U}{\partial r_{1} \partial r_{i} \partial r_{j}}\right)^{2}\right\rangle\right. \\
& \left.+k_{\mathrm{B}} T \sum_{i, j=1}^{3 N}\left\langle\frac{\partial^{2} U}{\partial r_{1} \partial r_{i}} \frac{\partial^{2} U}{\partial r_{1} \partial r_{j}} \frac{\partial^{2} U}{\partial r_{i} \partial r_{j}}\right\rangle\right]+O\left(\tau^{5}\right)
\end{aligned}
$$

whereas the equivalent expression for an atomic liquid is

$$
\begin{aligned}
\left\langle\Delta_{1}^{2}(\tau)\right\rangle= & \tau^{2} \frac{k_{\mathrm{B}} T}{m}-\frac{\tau^{4}}{12} \frac{k_{\mathrm{B}} T}{m^{2}}\left\langle\frac{\partial^{2} U}{\partial r_{1}^{2}}\right\rangle+\frac{\tau^{6}}{360} \frac{k_{\mathrm{B}} T}{m^{3}} \sum_{i=1}^{3 N}\left\langle\left(\frac{\partial^{2} U}{\partial r_{1} \partial r_{i}}\right)^{2}\right\rangle \\
& -\frac{\tau^{8}}{20160} \frac{1}{m^{4}}\left[3\left(k_{\mathrm{B}} T\right)^{2} \sum_{i, j=1}^{3 N}\left\langle\left(\frac{\partial^{3} U}{\partial r_{1} \partial r_{i} \partial r_{j}}\right)^{2}\right\rangle\right. \\
& \left.+k_{\mathrm{B}} T \sum_{i, j=1}^{3 N}\left\langle\frac{\partial^{2} U}{\partial r_{1} \partial r_{i}} \frac{\partial^{2} U}{\partial r_{1} \partial r_{j}} \frac{\partial^{2} U}{\partial r_{i} \partial r_{j}}\right\rangle\right]+O\left(\tau^{10}\right)
\end{aligned}
$$

These similarities and differences can be traced in part to the different equations of motion applying in the two cases. For a liquid Newton's law, equation (3.36), connects force and acceleration whereas in a particle suspension the force causes a coarse-grained 'drift' velocity,

$$
\bar{v}_{k}=-\frac{1}{\zeta} \frac{\partial U}{\partial r_{k}} .
$$

As mentioned above, the absence of odd powers of $\tau$ in the expansion of $\left\langle f_{1}(0) f_{2}(\tau)\right\rangle$ for a liquid reflects the time-reversibility of the motion. On the other hand the (coarse-grained) Smoluchowski description is not time reversible and both even and odd powers of $\tau$ are found in the expansion of $\left\langle f_{1}(0) f_{2}(\tau)\right\rangle$.

Finally, by comparing equations (3.24) and (3.59), we note that when hydrodynamic interactions are included in the description of a particle suspension the situation is considerably more complicated. This is because, then, not only do the interparticle forces depend on the instantaneous configuration of the particles (as in a liquid) but, in addition (and in contrast to the case of liquids), each particle's motion in response to these forces also depends on the positions of the particle through the configuration-dependent diffusion tensors. In this case the coarsegrained drift velocity is given by

$$
\bar{v}_{k}=\sum_{l=1}^{3 N}\left(\frac{\partial D_{k l}}{\partial r_{l}}-\beta D_{k l} \frac{\partial U}{\partial r_{l}}\right)
$$

which can be compared to equation (3.61) for the case when hydrodynamic interactions are neglected. Not surprisingly hydrodynamic interactions in a particle suspension (for which there is no equivalent in a simple liquid) lead to complicated dynamic many-body problem [36]. 


\subsection{Statistics of the particle displacement}

The intimate connection between the theory of gaussian random processes and the brownian motion of non-interacting particles has been recognized and exploited for many years. An expected effect of interparticle interactions is to introduce non-gaussian character into the displacements of particles occurring on timescales comparable to the structural relaxation time $\tau_{\mathrm{I}}$ described in $\S 1$.

Statistical properties of the particle displacement $\Delta_{1}(\tau)$ can be calculated by recognizing that the self intermediate scattering function, equation (3.33), can be viewed as a moment generating function for $\Delta_{1}(\tau)$, i.e.

$$
\begin{aligned}
F_{\mathrm{s}}(K, \tau) & \equiv\left\langle\exp \left[i K \Delta_{1}(\tau)\right]\right\rangle \\
& =\sum_{n=0}^{\infty} \frac{(i K)^{n}}{n !}\left\langle\left(\Delta_{1}(\tau)\right)^{n}\right\rangle .
\end{aligned}
$$

Alternatively a cumulant expansion gives

$$
F_{s}(K, \tau)=\exp \left[-K^{2} \rho_{1}(\tau)+K^{4} \rho_{2}(\tau)-K^{6} \rho_{3}(\tau)+\ldots\right]
$$

where the $\rho$ s are given by

$$
\left.\begin{array}{l}
\rho_{1}(\tau)=\frac{1}{2 !}\left\langle\Delta_{1}^{2}(\tau)\right\rangle \\
\rho_{2}(\tau)=\frac{1}{4 !}\left[\left\langle\Delta_{1}^{4}(\tau)\right\rangle-3\left\langle\Delta_{1}^{2}(\tau)\right\rangle^{2}\right] \\
\rho_{3}(\tau)=\frac{1}{6 !}\left[\left\langle\Delta_{1}^{6}(\tau)\right\rangle-15\left\langle\Delta_{1}^{4}(\tau)\right\rangle\left\langle\Delta_{1}^{2}(\tau)\right\rangle+30\left\langle\Delta_{1}^{2}(\tau)\right\rangle^{3}\right]
\end{array}\right\}
$$

etc.

If the displacement $\Delta_{1}(\tau)$ has gaussian statistics $\rho_{2}, \rho_{3}$ etc are all zero so that $\rho_{2}(\tau)$ provides the simplest measure of non-gaussian behaviour.

It has long been known [37] that for atomic liquids the leading term in $\rho_{2}(\tau)$ is of order $\tau^{8}$. This led to the conjecture [10] that for particle suspensions in which hydrodynamic interactions are neglected non-gaussian behaviour of the displacement would first be manifest as a $\tau^{4}$ term in $\rho_{2}(\tau)$. Previously [10] we calculated $F_{\mathrm{s}}(K, \tau)$ (for no hydrodynamic interactions) to order $\tau^{3}$; further calculation using equations (3.13) and (3.58) gives the $\tau^{4}$ term

$$
\begin{aligned}
\left\langle\exp \left(i K r_{1}\right)\right. & \left.\tilde{O}^{4} \exp \left(-i K r_{1}\right)\right\rangle=D^{4} K^{8}+6 \beta D^{4} K^{6}\left\langle\frac{\partial^{2} U}{\partial r_{1}^{2}}\right\rangle \\
& +D^{4} K^{4}\left\{4 \beta^{2} \sum_{i=1}^{3 N}\left\langle\left(\frac{\partial^{2} U}{\partial r_{1} \partial r_{i}}\right)^{2}\right\rangle+3 \beta^{2}\left\langle\left(\frac{\partial^{2} U}{\partial r_{1}^{2}}\right)^{2}\right\rangle-\beta\left\langle\frac{\partial^{4} U}{\partial r_{1}^{4}}\right\rangle\right\} \\
& +D^{4} K^{2}\left\{\beta^{2} \sum_{i, j=1}^{3 N}\left\langle\left(\frac{\partial^{3} U}{\partial r_{1} \partial r_{i} \partial r_{j}}\right)^{2}\right\rangle\right. \\
& \left.+\beta^{3} \sum_{i, j=1}^{3 N}\left\langle\frac{\partial^{2} U}{\partial r_{i} \partial r_{j}} \frac{\partial^{2} U}{\partial r_{1} \partial r_{i}} \frac{\partial^{2} U}{\partial r_{1} \partial r_{j}}\right\rangle\right\}
\end{aligned}
$$


Use of equations (3.59) and (3.63)-(3.66) then gives

$$
\rho_{2}(\tau)=\frac{\tau^{4}}{24} D^{4}\left\{3 \beta^{2}\left[\left\langle\left(\frac{\partial^{2} U}{\partial r_{1}^{2}}\right)^{2}\right\rangle-\left\langle\frac{\partial^{2} U}{\partial r_{1}^{2}}\right\rangle^{2}\right]-\beta\left\langle\frac{\partial^{4} U}{\partial r_{1}^{4}}\right\rangle\right\}+O\left(\tau^{5}\right)
$$

(no hydrodynamic interactions),

thus confirming the conjecture above.

A similar calculation can be made, with use of equations (3.34), (3.63) and (3.65), for the case when hydrodynamic interactions are taken into account. This gives

$$
\rho_{2}(\tau)=\frac{\tau^{2}}{2}\left[\left\langle D_{11}^{2}\right\rangle-\left\langle D_{11}\right\rangle^{2}\right]+O\left(\tau^{3}\right)
$$

(with hydrodynamic interactions).

Here we see that non-gaussian behaviour is evident at shorter times (to order $\tau^{2}$ ) than is the case when hydrodynamic interactions are neglected. Furthermore if, as in equation (2.9), we consider the particle displacement as composed of brownian and drift components we can show that the $O\left(\tau^{2}\right)$ non-gaussian behaviour in equation (3.68) arises entirely from the effect of hydrodynamic interactions on the brownian components of the particle displacements. Thus, adopting equations (2.9) and (2.23) complemented by the Itô prescription we write for the brownian component

$$
R_{1}(\tau)=\sum_{j=1}^{3 N} \int_{0}^{\tau} \sigma_{1 j}(t) f_{j}(t) d t
$$

where $\sigma_{i j}$ is the square root of the diffusion tensor defined by equation (2.24) and $f_{j}$ the white noise process defined by equations $(2.20)$ and $(2.21)$. To lowest order in $\tau$ we get

$$
\overline{R_{1}^{4}(\tau)}=\sum_{j, k, l, m=1}^{3 N} \sigma_{1 j} \sigma_{1 k} \sigma_{1 l} \sigma_{1 m} \int_{0}^{\tau} d t_{1} \int_{0}^{\tau} d t_{2} \int_{0}^{\tau} d t_{3} \int_{0}^{\tau} d t_{4} \overline{f_{j}\left(t_{1}\right) f_{k}\left(t_{2}\right) f_{l}\left(t_{3}\right) f_{m}\left(t_{4}\right)}
$$

Use of equations (2.20), (2.21) and (2.24) then gives

$$
\begin{aligned}
\widehat{R_{1}^{4}(\tau)} & =3 \sum_{j, l=1}^{3 N} \sigma_{1 j} \sigma_{1 j} \sigma_{1 l} \sigma_{1 l} 4 \tau^{2} \\
& =12 \tau^{2} D_{11}^{2}
\end{aligned}
$$

so that after ensemble averaging

$$
\left\langle\mathrm{R}_{1}^{4}(\tau)\right\rangle=12 \tau^{2}\left\langle D_{11}^{2}\right\rangle
$$

Then combining equations (3.24) and (3.70) gives

$$
\left\langle R_{1}^{4}(\tau)\right\rangle-3\left\langle R_{1}^{2}(\tau)\right\rangle^{2}=12 \tau^{2}\left[\left\langle D_{11}^{2}\right\rangle-\left\langle D_{11}\right\rangle^{2}\right],
$$

confirming that, to order $\tau^{2}$, non-gaussian behaviour of the total displacement (see equations (3.65) and (3.68)) arises entirely from the non-gaussian behaviour, 
caused by hydrodynamic interactions, of the brownian component of the displacement. By contrast, if hydrodynamic interactions are neglected

$$
\sigma_{i j}=D^{1 / 2} \delta_{i j}
$$

and it can be shown, by extending the calculations given above, that the brownian component of the displacement remains gaussian distributed at all times. In this case non-gaussian behaviour of the displacement is a consequence of the direct interparticle interactions. Of course, in the case where hydrodynamic interactions are important one would also expect non-gaussian behaviour due to the direct interactions but at higher order in $\tau$ than $\tau^{2}$.

As $D_{11} / k_{\mathrm{B}} T$ in equation (3.68) can be identified as a particle self mobility it is perhaps not surprising that the lowest order non-gaussian contribution to $\left\langle\Delta r_{1}^{2}(\tau)\right\rangle$ is simply proportional to the spread in mobilities $\left(\left\langle D_{11}^{2}\right\rangle-\left\langle D_{11}\right\rangle^{2}\right)$ that a particle experiences in all environments in the suspension. Explicit evaluation of equation (3.68) shows that the non-gaussian contribution is quite small in dilute suspension; however we might expect larger effects at higher concentration.

\section{Discussion and SUMmary}

A complete description of the dynamical properties of a particle suspension would, of course, require the solution of Newton's equations of motion for the $N$ brownian particles and the much larger number of liquid molecules. However, as outlined in $\S 1$, a knowledge of the time evolution of the probability distribution of particle positions is adequate to derive many useful properties of a suspension. Starting with the work of Einstein [3] this realization has motivated the development of coarse-grained descriptions of the suspension in which the detailed behaviour of the liquid is considerably 'averaged over'. Deriving these descriptions naturally involves approximations and a large literature exists on this subject. In this paper we do not address such matters but have essentially taken the Smoluchowski equation (2.4), which describes the evolution in time of the particle configuration space probability distribution, as the starting point for our discussion. What $Z$ wanzig [2] called the standard derivation of the Smoluchowski equation (a generalization of Einstein's treatment of non-interacting particles) already adopts a coarse-grained, diffusive picture of particle motions.

In $\S 2$ we have pointed out the equivalence of this Smoluchowski equation to, on the one hand, a truncated Kramers-Moyal expansion of the master equation and, on the other, a set of finite difference equations (2.9) for the particle trajectories. We then examined in some detail the passage from these finite difference equations to stochastic differential or Langevin equations and discussed the various forms of such equations which have appeared in the literature.

In $\S 3$ we considered the correlation function $F(\tau)$ (equation (3.1)) of variables $f_{1}$ and $f_{2}$ which are arbitrary functions of the particle positions. Using both the Smoluchowski ( $\$ 3.1$ ) and Langevin ( $\$ 3.2$ ) approaches we derived a formal expression (3.13) for the time evolution of $F(\tau)$ in a form which immediately lends itself to expansion in powers of the correlation delay time $\tau$. This result (3.13) has been obtained previously by several authors [31,32] who also pointed out the relevance of the adjoint Smoluchowski operator (equation (3.10)) in this context. In $\S 3.1$ we also wrote down explicit expressions for the first two terms in the time 
series expansion of $F(\tau)$; while the $\tau$ term (3.17) was given by Zwanzig [31] some time ago, the $\tau^{2}$ term (3.18) appears to be new.

We used these results to provide time series expansions to order $\tau^{2}$ of a particle's mean-square displacement and the self and full intermediate scattering functions $(\$ 3.3)$. One reason for calculating these series expansions is simply that it can, in principle, be done. In practice, the exact (within the limits of the Smoluchowski description) formal expressions for the coefficients of powers of $\tau$ quickly become prohibitively complicated with increasing order. Thus only shorttime $\left(\tau_{B} \ll \tau<\tau_{I}\right.$, in the nomenclature of $\S 1$ ) properties of the particle dynamics can be obtained rigorously by this approach. Nevertheless considerable effort has gone into evaluating the linear terms (related to effective diffusion coefficients which can be measured relatively easily by dynamic scattering methods) in these expansions for a variety of physical situations $[38,39]$. The $\tau^{2}$ term in equation (3.24) has been discussed recently $[11,40]$ for the case of a dilute suspension of ' hard-sphere' particles. In the simpler case where hydrodynamic interactions can be neglected, the first few terms in the power series expansions have also been used [41] to determine parameters in a memory function description of particle dynamics [32].

It is well known that correlation functions describing the dynamics of simple liquids can also be written as series in powers of $\tau$ (sometimes called 'sum rules') [42-44]. There are, in fact, formal similarities between the dynamics of atoms in a liquid and of particles in suspension, a realization which motivated some of the early suspension work $[30,34]$. In $\$ 3.4$ we explored these similarities (and differences) in more detail than hitherto by comparing the expansions of the mean-square displacements of an atom in a simple liquid and of a particle in a suspension in which hydrodynamic interactions are neglected.

In $\$ 3.5$, we investigated the statistical properties of the displacement of a particle in suspension by calculating its moments. We found that, due to interparticle interactions, the displacement is not generally a gaussian variable. Whereas direct interactions lead to non-gaussian behaviour at order $\tau^{4}$, hydrodynamic interactions cause it earlier, at order $\tau^{2}$.

P.N.P. and R.J.A.T. have benefited from helpful discussions with Dr A. J. Masters. The financial support of NATO, through research grant 132/84, is gratefully acknowledged.

\section{APPENDIX}

In this Appendix we discuss the relation between the Langevin equations proposed by $Z_{\text {wanzig }}[2]$ and Hess and Klein [8] to be equivalent to the Smoluchowski equation (2.4) within the Stratonovich interpretation. We also show explicitly that, while the Oseen tensor is divergenceless, the tensor whose square is equal to the Oseen tensor has a non-vanishing divergence.

Hess and Klein propose the Langevin equation (cf. equation (2.35))

$$
\frac{d r_{i}}{d t}=-\sum_{j} \beta D_{i j} \frac{\partial U}{\partial r_{j}}+\sum_{m, j} \sigma_{i m} \frac{\partial \sigma_{m j}}{\partial r_{j}}+\sum_{j} \sigma_{i j} f_{j}(t),
$$


where

$$
\sum_{m} \sigma_{i m} \sigma_{j m} \equiv D_{i j}
$$

while Zwanzig proposes

$$
\frac{d r_{i}}{d t}=-\sum_{j} \beta D_{i j} \frac{\partial U}{\partial r_{j}}-\sum_{m, j}\left(\frac{\partial}{\partial r_{j}} \sigma_{i m}\right) \sigma_{j m}+\sum_{j} \sigma_{i j} f_{j}(t) .
$$

To establish the identity of equations (A 1 ) and (A 3 ) in the Oseen approximation to $D_{i j}$ we note that, in this case,

$$
\frac{\partial}{\partial r_{j}} D_{i j}=0
$$

From equation (A 2) we now have

$$
\sum_{m}\left(\frac{\partial}{\partial r_{j}} \sigma_{i m}\right) \sigma_{j m}=-\sum_{m} \sigma_{i m} \frac{\partial}{\partial r_{j}} \sigma_{j m}
$$

substitution of this result into equation (A 3) and recognition of the symmetry property

$$
\sigma_{j m}=\sigma_{m j}
$$

demonstrate the equivalence of equations (A 3) and (A 2).

We now identify the tensor $\sigma$ whose square is the Oseen tensor, i.e. which satisfies

$$
\sigma(\mathbf{r}) \cdot \sigma(\mathbf{r})=\frac{1}{8 \pi \eta r}\left(1+\frac{\mathbf{r r}}{r^{2}}\right) .
$$

where 1 is the unit tensor. We make the ansatz

$$
\sigma(r)=\frac{1}{(8 \pi \eta r)^{1 / 2}}\left(a 1+b \frac{\mathbf{r r}}{r^{2}}\right)
$$

and find, by equating coefficients of 1 and $\mathbf{r r} / \mathbf{r}^{2}$, that

$$
a=1, \quad b=-1 \pm \sqrt{ } 2
$$

From equation (A 7) we see that

$$
\nabla . \sigma(\mathbf{r})=\frac{\mathbf{r}}{\left(32 \pi \eta r^{5}\right)^{1 / 2}}(3 b-a)
$$

and does not vanish.

\section{REFERENCES}

[1] Moyal, J. E., 1949, Ұ. R. Statist. Soc. B, 11, 150.

[2] Zwanzig, R., 1969, Adv. chem. Phys., 15, 325. An early discussion of the many particle Smoluchowski equation is given by Kirkwood, J. G., 1954, J. Polym. Sci., $12,1$.

[3] Einstein, A., 1956, Investigations on the Theory of Brownian Movement (Dover).

[4] Lax, M., 1966, Rev. mod. Phys., 38, 541.

[5] Mortensen, R. E., 1969, 尹. statist. Phys., 1, 271. 
[6] van Kampen, N. G., 1981, $\mathcal{~}$. statist. Phys., 24, 175.

[7] Lindenderg, K., Sheshadri, V., Schuler, K., and West, B. J., 1983, Probab. Analysis rel. Topics, 3, 18.

[8] Hess, W., and KLeIN, R., 1978, Physica A, 94, 71.

[9] Евмak, D. L., and McCammon, J. A., 1978, F. chem. Phys., 69, 1352.

[10] Pusey, P. N., and Tough, R. J. A., 1982, Y. Phys. A, 15, 1291.

[11] Pusey, P. N., and Tough, R. J. A., 1983, Faraday Discuss. chem. Soc., 76, 123.

[12] van Kampen, N. G., 1981, Stochastic Processes in Physics and Chemistry (NorthHolland), p. 102.

[13] However van Kampen, [12] p. 215, has stressed that the Kramers-Moyal result cannot be regarded as an expansion of the master equation in a power series in a small parameter.

[14] Middeten, D., 1960, An Introduction to Statistical Communication Theory (McGraw-Hill), Chap. 7.

[15] Wiener, N., 1923, $\mathcal{J}$. math. Phys., 2, 131.

[16] Schuss, Z., 1980, Theory and Applications of Stochastic Differential Equations (Wiley).

[17] GaRdiner, C. W., 1983, Handbook of Stochastic Methods (Springer).

[18] Dоов, J. L., 1942, Ann. Math., 43, 351, reprinted in: WaX, N. (editor), 1954, Selected Papers on Noise and Stochastic Processes (Dover).

[19] Rao, N. J., Borwankar, J. D., and Ramkrishna, D., 1974, S.I.A.M. Yl. Contr., $12,1$.

[20] [12], Chap. 8, §9.

[21] Jffrreys, H., and Jefrreys, B. S., 1972, Methods of Mathematical Physics (Cambridge University Press), p. $26 \mathrm{ff}$.

[22] Iтô, K., 1944, Proc. imp. Acad. Tokyo, 20, 519; 1951, Mem. Am. math. Soc., 4, 1.

[23] Stratonovich, R. L., 1963, Topics in the Theory of Random Noise, Vol. I (R. A. Silverman transl.) (Gordon \& Breach), Chap. 4, $\$ 8$.

[24] Wong, E., and ZAKaI, M., 1965, Int. J. engng Sci., 3, 213.

[25] Ryter, D., 1978, Z. Phys. B, 30, 219.

[26] See for example [7]. However the situation can be more intricate; see Graham, R., and Schenzle, A., 1982, Phys. Rev. A, 26, 1676.

[27] Arnold, L., 1973, Stochastische Differentialgleichungen (Oldenburg).

[28] Fox, R. F., 1972, Y. math. Phys., 13, 1196.

[29] Horsthemke, W., and Lefever, R., 1982, Noise-Induced Transitions (Springer).

[30] Ackerson, B. J., 1976, J. chem. Phys., 64, 242.

[31] Zwanzig, R., 1974, 9. chem. Phys., 60, 2717.

[32] Ackerson, B. J., 1978, 7. chem. Phys., 69, 684.

[33] [12], p. 129.

[34] Pusey, P. N., 1975, 7. Phys. A, 8, 1433.

[35] Hess, W., and Klein, R., 1983, Adv. Phys., 32, 173.

[36] Mazur, P., 1985, Can. Y. Phys., 63, 24.

[37] Schofield, P., 1961, Inelastic Scattering of Neutrons in Solids and Liquids (International Atomic Energy Agency), p. 39.

[38] Pusey, P. N., and Tough, R. J. A., 1985, Dynamic Light Scattering : Applications of Photon Correlation Spectroscopy, edited by R. Pecora (Plenum), p. 85.

[39] Schaefer, D. W., and Han, C. C., 1985, Dynamic Light Scattering : Applications of Photon Correlation Spectroscopy, edited by R. Pecora (Plenum), p. 181.

[40] Hinch, E. J., and Rallison, J. M., 1986, F. Fluid Mech., 167, 131.

[41] Arauz-Lara, J. L., and Medina-Noyola, M., 1986, J. Phys. A, 19, L117.

[42] De Gennes, P. G., 1959, Physica, 25, 825.

[43] Bansal, R., and Pathak, K. N., 1974, Phys. Rev. A, 9, 2773.

[44] Schofield, P., 1974, 7. Phys. A, 7, 246. 